and kills the $B$. coli, so that these first parasitic stages can be put into sterile media, of whatever composition the worker desires.

(10) More than two hundred media have so far been tried in attempts to cultivate these ex-sheathed larvæ further, but so far no method has been found of inducing them to cast the next sheath and so become fourth stage larvæ. Experimental work is needed to find out the conditions required for this.

It is impossible in a brief letter to indicate the innumerable problems which have arisen during the working out of this method, but I should be glad to supply further details to anyone interested. There are also many interesting problems for the study of which the 'adult' nematodes parasitic in sheep might be used, rather than such relatively specialised species as Ascaris.

GeOFFrey LAPAGE.

Institute of Animal Pathology, Cambridge, March 11.

'Wood, Second Rep. Direct. Inst. of Animal Path. Cambridge, 1931, p. 204.

'McCoy's method, Amer. J. Hyg., 11 ; 1930. An important paper, giving the results of its author's experimental work by modern methods on eggs and larvæ of Ancylostoma caninum.

\section{Anode Spot in a Neon Tube}

WHILE studying the phenomena of the moving striation and anode spot in a discharge tube filled with neon, our attention was directed to the fact that the anode spot is intermittent in its emission, with a rhythmic fluctuation in its intensity.

A photograph taken by a falling plate camera is reproduced in Fig. 1, while Fig. 2 shows another plate taken in a similar way but with the portion near the anode more magnified. In Fig. $1,+$ and - denote anode and cathode respectively, which were about $17.5 \mathrm{~cm}$. apart. The discharge tube was a straight one $4 \mathrm{~cm}$. in diameter. It was filled with pure neon at a pressure of a few centimetres and was excited

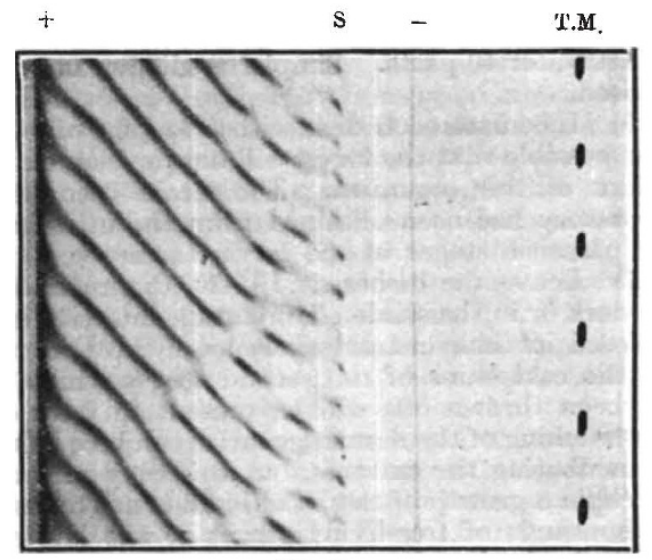

FIG. 1.

by a $2 \mathrm{kw}$. direct current generator giving 1,200 volts, the current in the tube being $190 \mathrm{ma}$. T.M. are the time marks, with an interval of $1 / 800 \mathrm{sec}$.

As will be seen in Fig. 2, the image of the anode spot, which was single in this case, is discontinuous in its emission. In fact, we see a series of luminous dots gradually increasing in its intensity, until, near the maximum intensity, one layer of the moving striation is detached from the anode and proceeds toward the cathode. The process is repeated at an interval of about $1 / 1160$ sec. In the case shown in Fig. 1 the velocity of the moving striation, at the middle of the positive column, is about 30 metres per second.

Another point of interest seen in Fig. 1 is the rhythmic fluctuation in the intensity of each of the moving striations. Apparently, the intensity of each stria decreases as it approaches the cathode, until it begins to appear on the plate as discontinuous strips. Near the end of the positive column, a small luminous sphere is seen which is detached from the uniform positive column. The luminosity of this sphere (marked S in Fig. 1) is also found to be intermittent, with an interval of $1 / 1880$ sec.

The remarkable regularity in the arrangement of

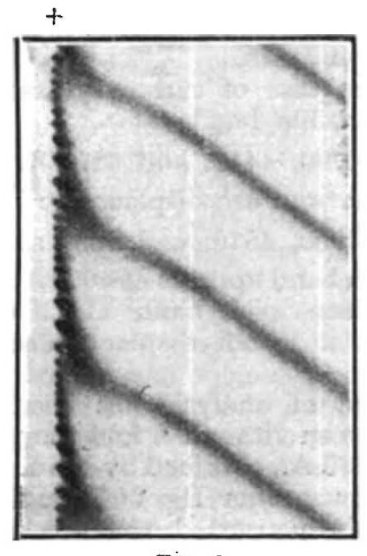

Fia. 2.

the strips in Fig. 1 would seem to suggest that there may be an interference between the moving striæ and some kind of disturbance regularly coming against it from the cathode.

As the anode spot is in fact the starting point of the moving striation, the closer investigation of its change with time may be of significance in the study of discharge through gases.

The details of the experiment will be published in the Scientific Papers of this Institute.

T. TAKAMINE.

T. SUGA.

A. YanaglHara.

Institute of Physical and Chemical Research, Komagome, Hongo-ku, Tokyo. Jan. 23.

\section{Rôle of the Solvent in Electrolytic Dissociation}

IN a recent paper ${ }^{1}$ Sir Harold Hartley and $\mathrm{Mr}$. O. L. Hughes bring forward evidence for the view that "solvation (of ions) is a statistical process, the relative amounts of the two kinds of molecules (in non-aqueous solutions containing small quantities of water) being roughly proportional to the concentrations in which they are present in the solution". This view is supported by the work of Butler ${ }^{2}$, who found that, although lithium chloride in alcohol-water mixtures is solvated preferentially by water, solvation by water is not an exclusive process.

Sir Harold Hartley goes on to interpret the degree of association of electrolytes in terms of (1) the size of the solvated ions, considered as rigid structures the motion of which obeys Stokes's law, and (2) the distance of approach of oppositely charged ions below which, according to Bjerrum's formula ${ }^{3}$, the number of ion-pairs (consisting of solvated ions) increases rapidly. 